\title{
The renaissance of acetaldehyde as a psychoactive compound: decades in the making
}

\author{
Mercè Correa $^{1 *}$, Elio Acquas ${ }^{2}$ and John D. Salamone ${ }^{3}$ \\ ${ }^{1}$ Department of Psicobiology, University Jaume I, Castelló, Spain \\ ${ }^{2}$ Department of Life and Environmental Sciences, University of Cagliari, Cagliari, Italy \\ ${ }^{3}$ Department of Psychology, University of Connecticut, Storrs, CT, USA \\ *Correspondence: correa@psb.uji.es
}

Edited and reviewed by:

Nuno Sousa, University of Minho, Portugal

Keywords: ethanol, catalase, acetate, dopamine, salsolinol, opioids, addiction, drug abuse

As with many events in the history of science, the development of the hypothesis that acetaldehyde is a plausible psychoactive substance with specific central effects (not related to its toxicity) has not been either incremental or progressive. Rather, it has evolved through a process of fits and starts. Initial clinical observations suggesting that accumulation of acetaldehyde could be used as a therapy for alcoholism did not lead to a highly effective treatment, and in fact, it was noted early on that small amounts of ethanol consumed under these conditions (i.e., blockade of aldehyde dehydrogenase) could be perceived as being even more pleasurable (Chevens, 1953). Although some laboratory data in animals appeared at that time (Carpenter and Macleod, 1952), it took a decade for the pre-clinical studies to focus on the potential importance of acetaldehyde. Since Myers proposed in the late 60 's that acetaldehyde could be a mediator of some of the effects of ethanol (Myers and Veale, 1969), advances in this field have gone through a push-pull process. His seminal discoveries about acetaldehyde self-administration and neural effects were met with skepticism because of the lack of replicability of results at that time, and the many unsolved questions related to measurements of brain acetaldehyde. In the late 70's, work conducted mainly by Amit's group (e.g., Amit et al., 1977), put acetaldehyde back in the picture. To replicate some of the early discoveries and expand the range of behaviors for which acetaldehyde could be shown to be a mediator of ethanol's effects, this laboratory started to assess the impact of interfering with acetaldehyde metabolism. Again, this work met with considerable skepticism, in part because of issues such as the lack of penetrability of acetaldehyde into the brain due to the high content of aldehyde dehydrogenase (ALDH) in the blood brain barrier, and also the absence of the alcohol dehydrogenase $(\mathrm{ADH})$ isoform that metabolizes ethanol in the brain (Lindros and Hillbom, 1979). More or less at that time, a newly characterized enzyme for the metabolism of ethanol was introduced to the cerebral enzymatic landscape; catalase (Cohen et al., 1980). This enzyme became the focus of the next wave of work by Aragon and Amit related to the acetaldehyde hypothesis (Aragon et al., 1985). In vitro and in vivo studies, mainly with one pharmacological tool and one genetic mutation, established the first plausible support for the idea that brain ethanol metabolism was possible, and had a clear impact on alcohol consumption (Aragon and Amit, 1993). In addition, late in the 90's the localization of high levels of catalase in catecholamine rich areas in the brain
(Zimatkin and Lindros, 1996), identified possible neuroanatomical loci for acetaldehyde formation. Across the first few years of the new century, Aragon and his colleagues developed a series of pharmacological tools that more firmly established the idea that brain catalase activity was directly correlated with ethanol's stimulant effects (Correa et al., 2001). Since then, data have been accumulating at an expanding pace. Additional groups of researchers, most of which are represented in this issue, started to apply different approaches to the "acetaldehyde hypothesis," which led to a new wave of papers and substantial scientific advances in this field. Genetic, neuropharmacological, electrophysiological and cellular mechanisms for acetaldehyde actions have been established. Potential therapeutic tools have been used. Neural circuits and complex behaviors have been studied, and other mediators of ethanol's actions that depend upon acetaldehyde formation, metabolism, or conjugation have been analyzed.

The present group of articles provides a comprehensive and up-to-date overview of research related to the actions of acetaldehyde, with recent results from the many groups that have decisively contributed to the field. The first review by Peana and Acquas gives a clear perspective on the behavioral evidence showing how acetaldehyde is a neuroactive molecule able to exert key motivational effects that can lead to the development of addiction, and also explains the neurobiochemical correlates of those actions in different intracellular signaling systems and diverse brain areas. A special accent on acetaldehyde formation, metabolism and blockade in relation to its reinforcing characteristics is presented by Diana and colleagues. Emphasis is put on the use of thiol compounds, which sequester acetaldehyde, as therapeutic agents preventing those effects. A very interesting methodological approach using gene-specific manipulations to change enzymatic activity, both in the liver and in the brain, has led Israel and colleagues to explore the behavioral consequences of central versus peripheral acetaldehyde metabolism. Direct administration of ethanol or acetaldehyde either in the periphery or in the brain, as employed in the studies from Correa and Salamone's lab, describe c-Fos expression (a marker of neural activity) across a broad range of brain areas involved in the motivational actions of both compounds. At the molecular level, Weiger and colleagues present data indicating that acetaldehyde acts on maxi calcium-activated potassium channels, a key factor in electrical stimulation of neural cells. 
The role of acetaldehyde/monoamine conjugates that form tetrahydroisoquinoline alkaloids is presented in the next group of articles. Special attention is paid to the conjugate of acetaldehyde with dopamine, salsolinol. This molecule has demonstrated to be central for the actions of ethanol in the mesolimbic system (Melis et al., 2013). Electrophysiological data presented by Xie, Ye and colleagues show how salsolinol modulates firing of dopamine neurons in brain slices from posterior ventral tegmental area via activation of opioid receptors located in neighboring GABAergic neurons. These data are discussed by Polache and Granero in a commentary, written from the perspective of their own extensive research on salsonilol-induced dopamine release in the accumbens in behaving animals. Deehan and Rodd, report on the role of salsonilol and other conjugates in acetaldehyde self-administration, in an integrative review of human findings and their own key animal data involving the mesolimbic dopaminergic system.

Although dopamine has been a major focus for studies of acetaldehyde's central actions, other neural systems are also involved. For instance, the role of the opioid system in the neurochemical and behavioral effects of acetaldehyde is reviewed by Font and Pastor, who not only discuss the effects of salsonilol on $m u$ opiod receptors, but also summarize what is known about acetaldehyde formation in brain nuclei rich in catalase and betaendorphine neurons. Elegant studies conducted by Cannizzaro's laboratory demonstrate that the endocannabinoid system modulates not only operant acetaldehyde self-administration, extinction, and relapse, but also its effects on the emission of punished responses during conflict experiments, all of which are key features of addiction. A relevant role for acetate, a metabolite of acetaldehyde, as a mediator of the sedative and ataxic effects of alcohol is presented by Correa and colleagues. These results are hypothesized to be mediated by the neuromodulator adenosine.

The work by March, Molina and colleagues is focused on a particulary important neurodevelopemental period; early ontogeny. At this stage, drugs have a substantial impact on the nervous system, and in the case of ethanol, its actions can be affected by the fact that rats show a high rate of brain catalase activity specifically in this period. Thus, these authors provide extensive results about vulnerability to abuse and addiction in newborn and infant rats, which is induced by early ethanol and acetaldehyde exposure. These data are discussed in a commentary by Zimatkin, an expert on brain enzymatic localization, in terms of the ideal scenario in brain pups for acetaldehyde accumulation (high catalase-low ALDH). Finally, on the other extreme of the neurodevelopmental process, Vaglini, Corsini and colleagues review data on the impact of acetaldehyde on Parkinson's desease. In this case the authors focus on the role of the other important brain enzymatic system for the central formation and degradation of acetaldehyde; cytochrome P450-2E1, which is highly concentrated in substantia nigra pars compacta, an area rich in dopamine cell bodies. Acetaldehyde acting as a substrate/inhibitor of this enzyme, may facilitate the susceptibility of these dopaminergic neurons to toxin-induced parkinsonism.

Many more exciting discoveries are yet to come, and new methodologies still need to be developed in order to overcome the obstacles that continue to obscure our understanding of the role of acetaldehyde. Especially important would be the need for reliable measurements of central acetaldehyde production under physiological conditions. However, we can certainly say that acetaldehyde, its metabolites and its conjugates are critical molecules for the complex set of effects observed after ethanol consumption, especially those related to the development of its pathological consumption.

\section{ACKNOWLEDGMENTS}

Mercè Correa received support from Plan Nacional de Drogas (2010I024), Spain. John D. Salamone was supported by NIH/NIMH (MH078023-01A1), USA. Elio Acquas received grants from the Italian Ministry of University and Research (MIUR, PRIN 2006), and the Regione Autonoma della Sardegna (RAS), Italy, Regional Law $\mathrm{n}^{\circ} 7 / 2007, \mathrm{n}^{\circ} \mathrm{F} 71 \mathrm{~J} 090006200002$ and B71J11001480002_1.

\section{REFERENCES}

Amit, Z., Brown, Z. W., and Rockman, G. E. (1977). Possible involvement of acetaldehyde, norepinephrine and their tetrahydroisoquinoline derivatives in the regulation of ethanol seld-administration. Drug Alcohol Depend. 2, 495-500.

Aragon, C. M., and Amit, Z. (1993). Differences in ethanol-induced behaviors in normal and acatalasemic mice: systematic examination using a biobehavioral approach. Pharmacol. Biochem. Behav. 44, 547-554. doi: 10.1016/00913057(93)90165-P

Aragon, C. M., Sternklar, G., and Amit, Z. (1985). A correlation between voluntary ethanol consumption and brain catalase activity in the rat. Alcohol. 2, 353-356. doi: 10.1016/0741-8329(85)90074-6

Carpenter, R. K., and Macleod, L. D. (1952). The effects of ethyl alcohol and acetaldehyde on maze behaviour and motor co-ordination in rats. J. Ment. Sci. 98, 167-173.

Chevens, L.C. F. (1953). Antabuse addiction. Br. J. Med. 1, 1450-1451. doi: 10.1136/bmj.1.4825.1450-c

Cohen, G., Sinet, P. M., and Heikkila, R. (1980). Ethanol oxidation by rat brain in vivo. Alcohol Clin. Exp. Res. 4, 366-370.

Correa, M., Sanchis-Segura, C., and Aragon, C. M. (2001). Brain catalase activity is highly correlated with ethanol-induced locomotor activity in mice. Physiol. Behav. 73, 641-647. doi: 10.1016/S0031-9384(01)00511-X

Lindros, K. O., and Hillbom, M. E. (1979). Acetaldehyde in cerebrospinal fluid: its near-absence in ethanol-intoxicated alcoholics. Med. Biol. 57, 246-247.

Melis, M., Carboni, E., Caboni, P., and Acquas, E. (2013). Key role of salsolinol in ethanol actions on dopamine neuronal activity of the posterior ventral tegmental area. Addict. Biol. doi: 10.1111/adb.12097. [Epub ahead of print].

Myers, R. D., and Veale, W. L. (1969). Alterations in volitional alcohol intake produced in rats by chronic intraventricular infusions of acetaldehyde, paraldehyde or methanol. Arch. Int. Pharmacodyn. Ther. 180, 100-113.

Zimatkin, S. M., and Lindros, K. O. (1996). Distribution of catalase in rat brain: aminergic neurons as possible targets for ethanol effects. Alcohol Alcohol. 31, $167-174$.

Conflict of Interest Statement: The authors declare that the research was conducted in the absence of any commercial or financial relationships that could be construed as a potential conflict of interest.

Received: 05 June 2014; accepted: 01 July 2014; published online: 22 July 2014. Citation: Correa M, Acquas E and Salamone JD (2014) The renaissance of acetaldehyde as a psychoactive compound: decades in the making. Front. Behav. Neurosci. 8:249. doi: 10.3389/fnbeh.2014.00249

This article was submitted to the journal Frontiers in Behavioral Neuroscience. Copyright (c) 2014 Correa, Acquas and Salamone. This is an open-access article distributed under the terms of the Creative Commons Attribution License (CC BY). The use, distribution or reproduction in other forums is permitted, provided the original author(s) or licensor are credited and that the original publication in this journal is cited, in accordance with accepted academic practice. No use, distribution or reproduction is permitted which does not comply with these terms. 